\title{
Analysis of impact cam mechanisms from the point of view of the rationality of their use in drilling machines
}

\author{
Anna Teplyakova ${ }^{1}$, Ivan $_{\text {Zhukov }}^{1, *}$ \\ ${ }^{1}$ Saint Petersburg Mining University, 2, 21st Line, St. Petersburg 199106, Russia
}

\begin{abstract}
Modern drilling machines designed for the destruction of rocks have a diverse structure, various performance characteristics and in most cases a well-defined limited scope of application. One of the variants of the design of such machines is the integration of impact cam mechanisms into their structure, the variety of which allows achieving the specified working conditions. The most suitable one for impact machines is the front cam mechanism, in which the rotational movement of the cam is converted into the translational movement of the driven link - the pusher. Only cam mechanisms allow implementing a certain law of motion of the output-working link accurately. This article provides an overview of the well-known patented impact machines equipped with impact cam mechanisms. A brief description of each technical solution is provided, indicating mechanism differences and uniqueness. Based on the results of the critical analysis of the found machines and mechanisms, the tasks of further research are formulated. These results will be the development of scientific knowledge in the field of the use of cam mechanisms in impact machines, namely in drilling machines that can automatically adapt to operating conditions.
\end{abstract}

\section{Introduction}

One of the main requirements for modern machines is the relevance to ensure the movement of the output links according to a given law of motion. Simple methods of execution and connection of parts do not allow this to be achieved. The implementation of this condition is possible through the use of links having a curved working surface. The most common example of such a link is a cam that forms a higher kinematic pair with the output link.

Cam mechanisms have a number of advantages. The process of designing them is quite simple. The cam profile can be set to any geometry, making it easy to adjust to different requirements. Such mechanisms make it possible to implement the specified law of motion of the driven link with greater ease, accuracy and economy.

The field of application of cam mechanisms is very extensive and includes mining, metalworking, printing and other machines. In mining machines of impact action, the cylindrical front cam mechanism has become predominant. It is used mainly as a drive mechanism that provides the cocking of the anvil block by turning the cam to influence a pillar or tool.

A cylindrical front cam mechanism (Figure 1) is usually called a spatial mechanism in which the driving link (cam) performs a rotational movement, the driven link moves rectilinearly and translationally (pusher), and the axis of the pusher is parallel to the axis of rotation of the cam.

A detailed study of cam profile, graphs of the speeds and accelerations of pusher allows getting an idea of the interrelation between cam profile geometry, stress, dynamic loads, noise and vibration in the machine elements.

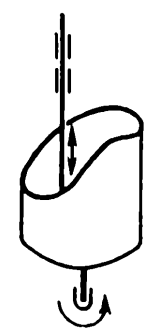

Fig. 1. Front cam mechanism with roller pusher.

In most cases, such mechanisms use a roller pusher equipped with a cylindrical or conical roller. The main disadvantage of such a mechanism is that the cam profile is too steep and can lead to cramping the forwardmoving pusher. For proper operation of the mechanism, the pusher must be pressed against the cam at all speeds. One of the options for ensuring this condition is the use of a connecting spring.

\section{Solution methods}

The scientific research of various scientists, both in Russia and abroad, is devoted to the analysis and synthesis of drilling machines equipped with cam mechanisms. According to the results of the patent search, it was found that the most active work in this direction was carried out in the National Academy of Sciences of the Kyrgyz Republic and Novosibirsk State Technical University. One of the first such drilling

\footnotetext{
Corresponding author: tmmiok@yandex.ru
} 
machines was patented in 1950; their further spread was due to the development of spacecraft, in particular, designed for drilling and taking soil from the Moon. This direction was supervised by Academician Oleg Dmitrievich Alimov, who together with his colleagues managed to create and test series of similar machines. In parallel, impact machines equipped with a cam mechanism, designed for peaceful purposes, begin to appear, specifically in the perforator construction. The most significant results in this direction were obtained by Professor Anatoly Yarunov.

Let us turn in more detail to the well-known patented drilling machines with impact cam mechanisms (Table 1), each of which can find a rational application for performing a certain type of work on the destruction of rock.

Table 1. Patented drilling machines with a cam mechanism.

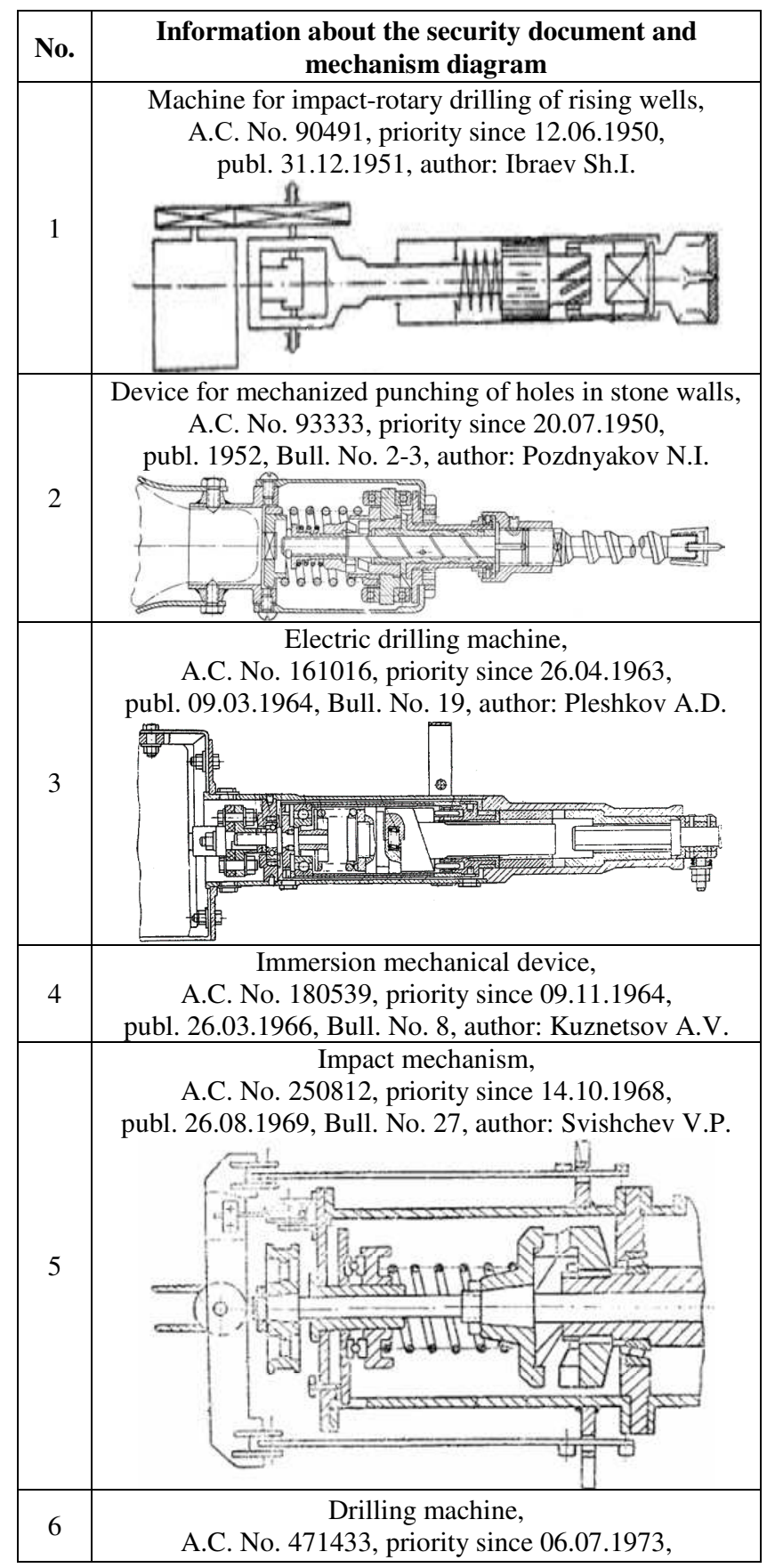

publ. 25.05.1975, Bull. No. 19, authors: Alimov O.D., Manzhosov V.K., Shevchenko B.A.

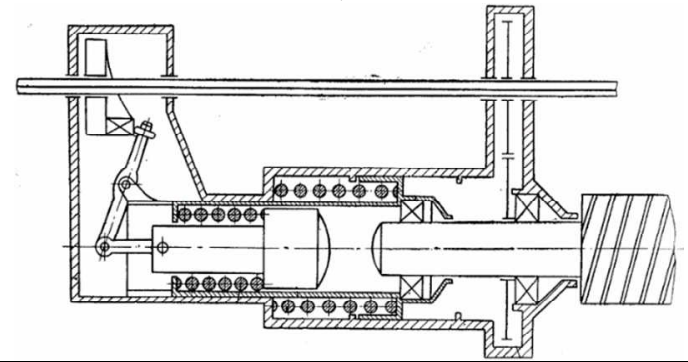

Drilling machine,

A.C. No. 481694 , priority since 29.06 .1973 , publ. 25.08.1975, Bull. No. 31, authors: Izyumin Yu.A., Eliseev V.G., Prokhorov A.A., et al.

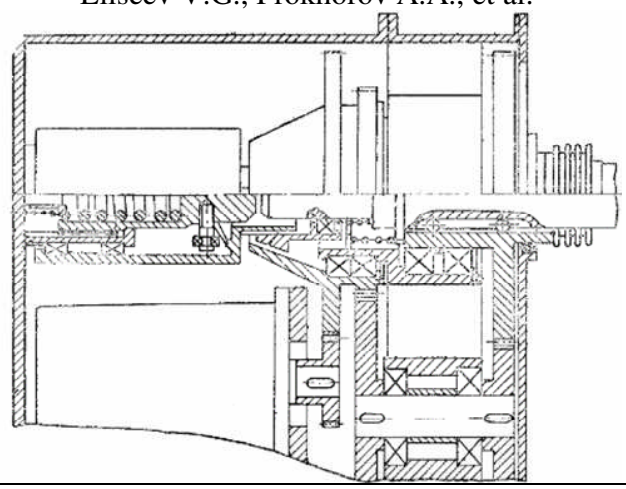

Electric drilling machine,

A.C. No. 548709 , priority since 06.07 .1973 , publ. 28.02.1977, Bull. No. 8, authors: Alimov O.D., Manzhosov V.K., Shevchenko B.A.

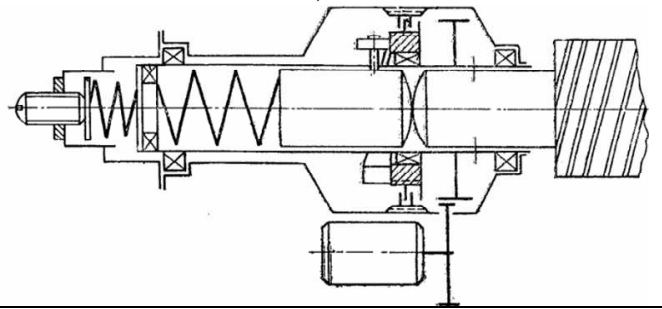

Electric drilling machine,

A.C. No. 570704 , priority since 16.01 .1975 , publ. 30.08.1977, Bull. No. 32, authors: Alimov O.D.,

Manzhosov V.K., Shevchenko B.A.

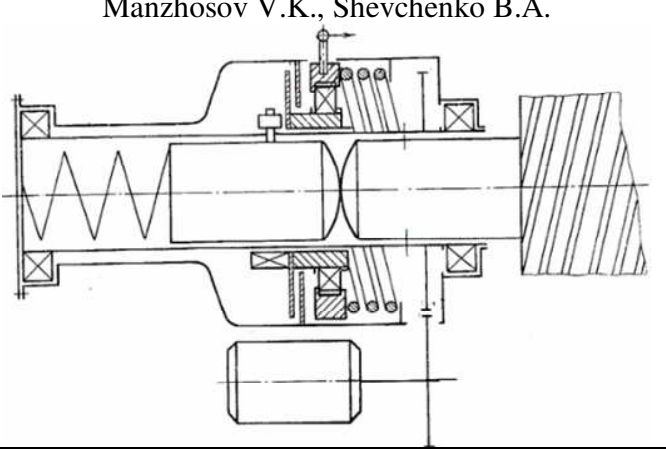

Immersion device for impact-rotational drilling,

A.C. No. 578451, priority since 17.06 .1975 , publ. 30.10.1977, Bull. No. 40, authors: Alimov O.D., Manzhosov V.K., Shevchenko B.A., Voronin Yu.K.

10

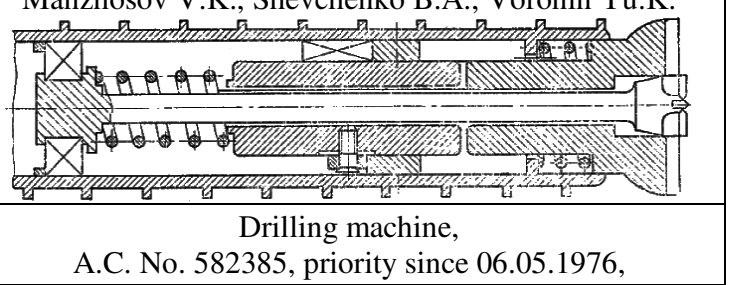



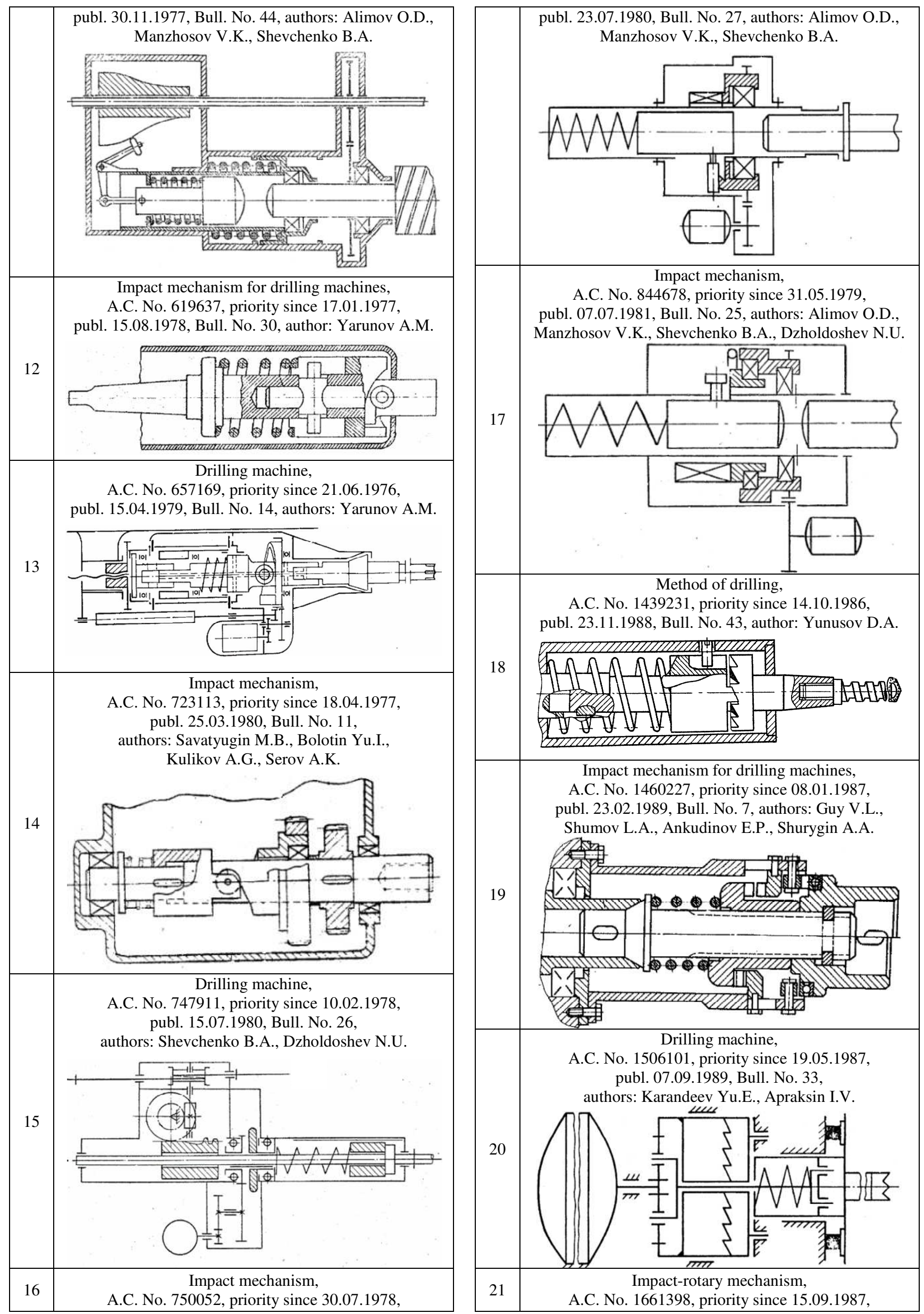


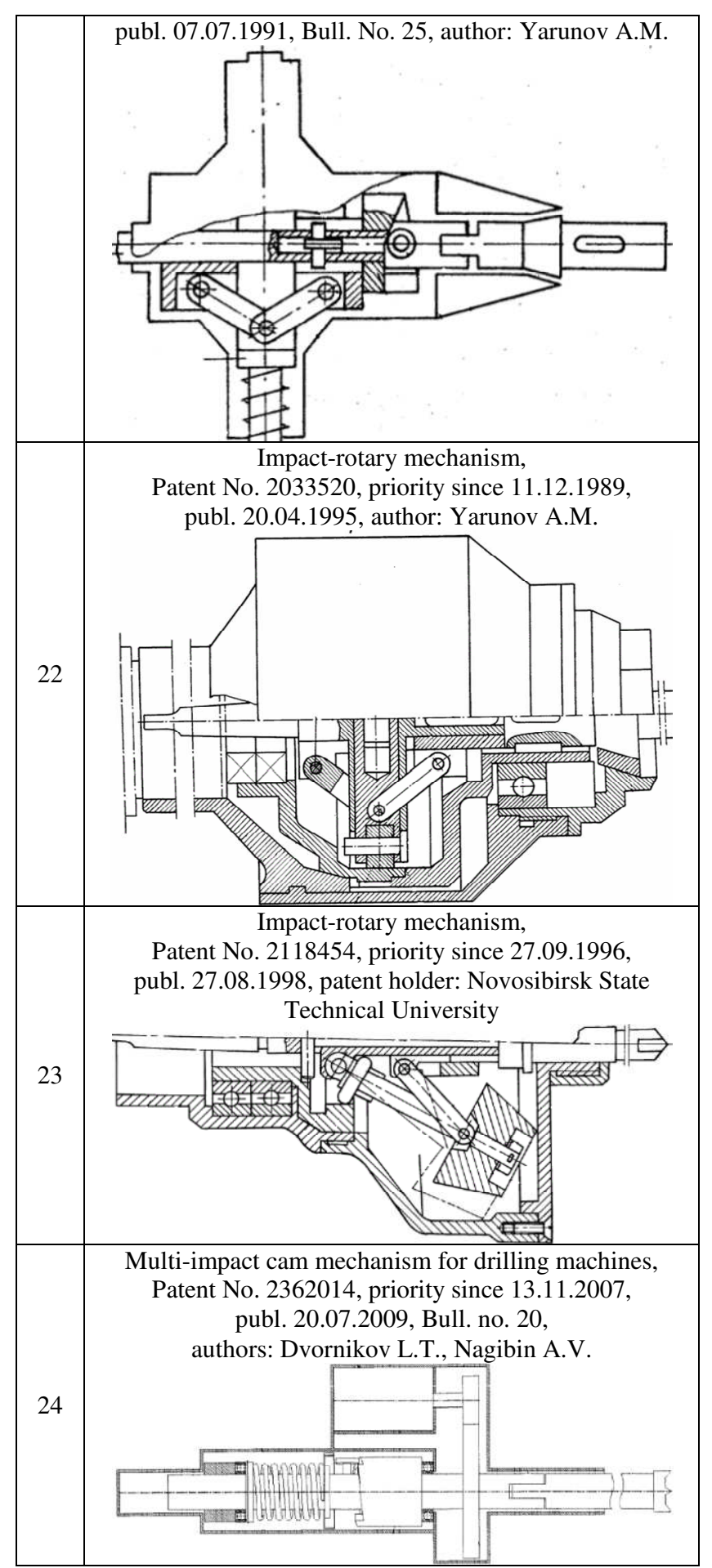

One of the first drilling devices equipped with a cam mechanism was patented in the 50s of the last century, a machine (No. 1, Table 1) for impact-rotary drilling of rising wells, which is most effective for working on strong rocks. The machine uses a spring-loaded impact pillar, which is tightened to influence the bit by means of a disk cam located in the slot of the pillar shank, which rotates due to an electric motor.

At the same time, we created a device (No. 2, Table 1) for mechanized punching holes in stone walls. The device is mounted on an electric drill in order to provide forced strokes of the pillar on the destroyed object. The rod of the electric motor is coupled through a spring with a bearing washer, which, running around the cam profile, provides a blow to the destroyed object. The strokes alternate with the rotation of the rod, and the number of strokes is determined by the product of the speed of the drill by the number of cams. The part, called by the authors a cam washer, is essentially a cylindrical front cam, while ball bearings are used as rolling rollers on it. The development of such an impact mechanism was an electric drilling machine (No. 3, Table 1), which uses a similar impact cam mechanism. The difference of the invention lies in the installation of special shockabsorbing springs in the cams, which make it possible to extinguish the impact and vibration of the rollers at the moment of their contact with the cam.

The first use of cam mechanisms in immersion impact machines is recorded by the author's certificate (No. 4, Table 1), published in 1996. In the invented device, two axially arranged cam systems are used; they transmit a blow and a turn to the drilling tool. The first system is positively connected to the frame, while the cams of the second system are placed between the cams of the first system on a hollow rod positively connected to the drilling tool, on which the rollers are fixed, serving for cocking the impact system. Unfortunately, the device diagram is not preserved.

The most urgent task in the development of drilling machines is to ensure the automatic selection of operating modes depending on the operating conditions. One of the variants of the mechanism that implements this task is the impact mechanism according to the author's certificate No. 250812 (No. 5, Table 1), which allows one to turn on and off the impact function automatically. This is achieved through the use of cams with ratchet teeth, the presence of contact between which depends on the value of the force feed of the drilling tool.

In 1975, a team of authors from the Academy of Sciences of the Kyrgyz SSR created a drilling machine for drilling holes and boreholes in rocks of various strength (No. 6, Table 1), in which the anvil block of the impact system is cocked using a front cam. When drilling a rock of small strength, the contact of the roller connected to the anvil block and the cam is absent, the anvil block is not cocked, and the machine operates in the rotary drilling mode. With an increase in the strength of the rock, the spring is compressed, which prevents the contact of the roller and the cam. It leads to the lifting of the anvil block and the inclusion of the impact mechanism in the work - the machine begins to work in the mode of impact-rotational drilling.

Further for the same purpose - automatic control of drilling modes depending on the strength of the rock was proposed as the design of the drilling machine (No. 7, Table 1), in which the inclusion of a cam mechanism that provides an impact effect on the destroyed object occurs when the rock strength increases

For the same purpose - automatic control of drilling modes depending on the strength of the rock, the design of the drilling machine was proposed (No. 7, Table 1), in which the inclusion of a cam mechanism that provides a shock effect on the destroyed object occurs when the rock strength increases. The implementation of the idea 
of automatic selection of the operating mode is provided by a bushing movable in the axial direction that turns on and off the rotation of the cam against the resistance force of the rock to the introduction of the tool.

According to the same principle of ensuring automatic change of the rotary mode of operation of the drilling machine to the impact-rotary mode, the mechanisms listed in Table 1 under numbers No. 8-11 were designed, including for immersion drilling (No. 10, Table 1).

The research cycle, which resulted in drilling machines according to the inventions listed under numbers $6,8-11$ in Table 1 , was based on the government task of the USSR related to drilling and soil sampling on the Moon.

The next version of the technical solution for the use of a cam mechanism in a drilling machine was the mechanism of A.M. Yarunov (No. 12, Table 1), in which the cam is used not only for cocking the impact body, but is also for the drummer itself. This design allows you to simplify the design of the machine and reduce the complexity of manufacturing its elements. Further research by the author led to the development of a new design (No. 13, Table 1), in which the front cam has only a rotational movement, which is converted by the mechanism into a forward movement of the anvil block, equipped with rollers that roll around the cam. A special feature of this invention was the possibility of providing the necessary amount of lubrication of the machine elements and eliminating the impact on the drive motor.

In order to increase the energy of a single impact, there was an impact mechanism in 1980 (No. 14, Table 1), in which the shank of the drilling tool and the anvil block rotate with the same angular velocity, while due to the built-in front cam, the spring is cocked. This provides an impact with the anvil block on the shank. The mechanism differs from the known ones because besides the roller there is an additional front projection interacting with the cam, it is located perpendicular to the axis of the anvil block at the level of the tangent to the circumference of the side surface of the roller. The presence of such a projection allows the anvil block not to smoothly roll down the profile of the cam, but to break off instantly, thereby increasing the impact energy.

The work of the staff of the Academy of Sciences of the Kyrgyz SSR was continued using several designs of drilling machines with cam impact mechanisms (No. 1517, Table 1). Their device provides not only automatic selection of a particular drilling mode, but also allows one to adjust the tool force feed depending on the strength of the destroyed rock. Moreover, only in one of the named inventions (No. 16, Table 1) the authors pay attention to the geometry of the cam profile, justifying its rationality by the constant moment of loading the drive, determined by the formula:

$$
M=P \cdot r_{k} \cdot \operatorname{tg} \alpha,
$$

where $P$ is the cocking force of the anvil block, determined by the characteristic of the preload spring, $r_{k}$ is the radius of the profile part of the cam, $\alpha$ is the angle of lifting of the cam profile.

Constant value of the moment $M$ at different values of the anvil block cocking force is provided by the selection of variable values of the profile radius and the cam lifting angle, which allows one to increase the efficiency of the device generally.

In 1988, a method for drilling rocks was patented (No. 18, Table 1); it is based on the use of a rockbreaking machine, the structure of which includes two interacting cams. Their profiles are made in the form of a toothed or ratchet surface. This design of the machine provides an impact-rotary drilling mode with a certain law of alternation of impact and rotation of the tool, which allows to impact only on the untreated surface of the bottom face with a cutting tool. It helps to reduce tool wear and avoid over-grinding of destruction products.

A similar geometry of the cam profile is used in the design of the drilling machines according to the author's certificates No. 1460227 (No. 19, Table 1) and No. 1506101 (No. 20, Table 1). This version of the cams allows an automatic increase in the impact axial load on the face with an increase in the resistance of the destroyed rock to the introduction of the drilling tool or completely disable the impact mode when destroying materials of small strength.

The next series of inventions of impact machines (No. 21-23, Table 1), equipped with cam mechanisms, were proposed by A.M. Yarunov, who, in addition to the front cam, began to use a disk cam, justifying the replacement by increasing the reliability of the mechanism and reducing manufacturing costs. At the same time, he introduces several lever links into the structure of the machine, the using of which is designed to generate impact energy.

One of the latest, protected by a patent for the invention, is a multi-impact mechanism of a drilling machine (No. 24, Table 1), which is equipped with a cam with several sections of the anvil block cocking, moreover the sections of the cocking are arranged successively and differ from each other in the height of the profile. It leads to a change in the stroke of the anvil block from section to section of the cocking. This version of the cam impact mechanism allows changing the effect of the impact of the drilling tool on the face and thereby provides an increase in the productivity of drilling operations.

\section{Results and discussion}

The analysis of the known design versions of machines with impact cam mechanisms allowed establishing the main objectives of the study of cam mechanisms, the solution of which will increase the efficiency of rock destruction by drilling machines.

1. Selection and justification of the geometric parameters of the working profile of the cam, which provides the maximum efficiency of the mechanism. Of all the inventions listed in Table 1, only one is intended to increase the efficiency of the device. The way to achieve this goal is based on ensuring the constant loading of the drive. However, in the general case, the efficiency of the machine is determined by the ratio of useful work to spend. The main purpose of the cam 
mechanism used in impact machines is the cocking of the striking body. The useful work of the mechanism is defined as the work of the force $F$ of the impact by the cam on the pusher-striker on a certain path $\mathrm{s}$ of the point of contact of the driven link with the cam:

$$
A=\int_{s} F \cdot \cos \lambda d s
$$

where the angle $\lambda$ is the pressure angle formed between the normal to the cam profile with the direction of the vector of the absolute linear velocity of the pusherstriker.

The magnitude of the force $F$, which the cam is directed to overcome, is determined by the characteristic of the preload spring, and the pressure angle $\lambda$ directly depends on the geometry of the cam profile. The working profile of the cam is set on its sweep and is determined by the type of line used. Mainly, a straight line is taken; its characteristic feature is a constant value of the pressure angle. This solution does not allow solving the problem of ensuring maximum useful work. Therefore, finding and analyzing the curve of the line forming the cam profile will allow to achieve an increase in the efficiency of the mechanism.

The solution of this task has another important goal. At the point of contact of the roller with the pusherstriker with the cam, the force of their interaction is decomposed into two components. One of them is directly aimed at lifting the pusher. Moreover, the second causes the roller to slip and prevents the cam from turning, which can lead to jamming of the mechanism. In this regard, the task of finding such a curve describing the cam profile, which will ensure a reduction in the power load that prevents the normal operation of the mechanism, is very relevant.

2. Construction and analysis of a mathematical model of the impact process in the system of a drilling machine with a cam impact mechanism. The solution of this problem will allow establishing functional relationships between the impact energy, the height and the number of sections of the cocking profile of the cam accordingly to the mechanical properties of the destroyed object. Among the known only two cam mechanisms (Nos. 2 and 24, Table 1), implemented in mining machines of impact action, provide the application of several strikes with a striker when turning the cam for one turn. The devices listed in Table 1 under numbers 18-20 produce several strokes on the tool in one turn, thereby providing a rotary-impact drilling mode. But in fact, they are unsuitable for the destruction of high-strength rocks, which are should be drilled in the mode of impact with turn through the higher value of the energy of a single impact. Matching the dependence of the resistance force of the rock to the introduction of the tool with the impact energy due to the parameters of the cocking sections of the cam profile will minimize energy losses performing work on the destruction of rock by impact.

3. Development and research of adaptive drilling machine. When drilling rock in difficult geological conditions, there is naturally a need to destroy objects of different strength. In this case, the effective operation of the same machine is possible only if it is automatically adapted to the conditions of drilling operations. The development of such machines is the research of various scientists, the results of which were the majority of the inventions analyzed in this article. However, it is worth noting that the scope of application of these machines is still limited to a certain range of values of the strength coefficient, i.e. they do not allow implementing the mode of applying powerful impacts with periodic tool rotation required for the destruction of high-strength rocks. In this regard, the task of finding design solutions of complex gear-cam mechanisms that allow one to adapt the drilling machine to various operating conditions by automatically selecting rotary, rotaryimpact, impact with turn or impact drilling is undoubtedly relevant.

The solution of the formulated tasks will allow one to develop the theoretical foundations of the design of impact cam mechanisms, to develop scientific and methodological foundations for the creation and research of impact systems of adaptive drilling machines designed for the destruction of rocks of a wide range of values of the strength coefficient.

\section{References}

1. L.N. Reshetov, Cam mechanisms, (Moscow: Mashgiz, 1953)

2. N.N. Popov, Calculation and design of cam mechanisms, (Moscow: Mechanical Engineering, 1980)

3. H.A. Rothbart, Cam design handbook, (New York: McGraw-Hill Professional Publ., 2003)

4. A.M. Yarunov, Cam mechanisms of variable structure, (Irkutsk: Irkutsk University Press, 1986)

5. L. Faxin, F. Xianzhang, Procedia Environmental Sciences, 10, 1343-1349 (2011), doi: 10.1016/j.proenv.2011.09.215.

6. C. Zhoua, B. Hua, S. Chenb, L. Mac, Mechanism and Machine Theory, 104, 118-129 (2016), doi: 10.1016/j.mechmachtheory.2016.05.009.

7. N. Sateesh, C.S.P. Rao, T.A. Janardhan Reddy, International Journal of Computer Integrated Manufacturing, 22(6), 515-523 (2009), doi: 10.1080/09511920802546814.

8. J. Ondrášek, Procedia Engineering, 92, 320-329 (2014), doi: 10.1016/j.proeng.2014.12.129.

9. A.V. Nagibin, L.T. Dvornikov, Mining equipment and electromechanics, 1, 34-40 (2013)

10. Sh.I. Ibraev, A.C. No. 904914 (USSR), Machine for shock-rotary drilling of rising wells, (31.12.1951)

11. N.I. Pozdnyakov, A.C. No. 93333 (USSR), Device for mechanized punching holes in stone walls, (20.07.1950)

12. A.D. Plechkov, A.C. No. 161016 (USSR), Electric drilling machine, (09.03.1964)

13. A.V. Kuznetsov, A.C. No. 180539 (USSR), Submersible mechanical device, (26.03.1966)

14. V.P. Svishchev, A.C. No. 250812 (USSR), Impact mechanism, (26.08.1969) 
15. O.D. Alimov, V.K. Manzhosov, B.A. Shevchenko, A.C. No. 471433 (USSR), Drilling machine, (25.05.1975)

16. Y.A. Izyumin, V.G. Eliseev, A.A. Prokhorov, S.V. Dmitriev, Yu.N. Yudin, V.V. Andryushchenko, P.V. Pshenichnij, A.C. No. 481694 (USSR), Drilling machine, (25.08.1975)

17. O.D. Alimov, V.K. Manzhosov, B.A. Shevchenko, A.C. No. 548709 (USSR), Electric drill machine, Ddecl. (28.02.1977)

18. O.D. Alimov, V.K. Manzhosov, B.A. Shevchenko, A.C. No. 570704 of the (USSR), Electric drill machine, (30.08.1977)

19. O.D. Alimov, V.K. Manzhosov, B.A. Shevchenko, Yu.K. Voronin, A.C. No. 578451 (USSR), Submersible device for percussive-rotary drilling, (30.10.1977)

20. O.D. Alimov, V.K. Manzhosov, B.A. Shevchenko, A.C. No. 582385 (USSR), Drill machine, (30.11.1977)

21. A.M. Yarunov, A.C. No. 619637 of the (USSR), Impact mechanism for drilling machines, (15.08.1978)

22. A.M. Yarunov, A.C. No. 657169 (USSR), Drilling machine, (15.04.1979)

23. M.B. Savatyugin, Yu.I. Bolotin, A.G. Kulikov, A.K. Serov, A.C. No. 723113 (USSR), Impact mechanism, (25.03.1980)

24. B.A. Shevchenko, N.U. Dzholdoshev, A.C. No. 747911 (USSR), Drilling machine, (15.07.1980)

25. O.D. Alimov, V.K. Manzhosov, B.A. Shevchenko A.C. No. 750052 (USSR), Impact mechanism, (23.07.1980)

26. O.D. Alimov, V.K. Manzhosov, B.A. Shevchenko, N.U. Dzholdoshev, A.C. No. 844768 (USSR), Impact mechanism, (07.07.1981)

27. D.A. Yunusov, A.C. No. 1439231 (USSR), Method of drilling, (23.11.1988)

28. V.L. Goy, L.A. Shumov, E.P. Ankudinov, A.A. Shurygin, A.C. No. 1460227 (USSR), Impact mechanism for drilling machines, (23.02.1989)

29. Yu.E. Karandeev, I.V. Apraksin, A.C. No. 1506101 (USSR), Drilling machine, (07.09.1989)

30. A.M. Yarunov, A.C. No. 1661398 (USSR), Impactrotary mechanism, 0(7.07.1991)

31. A.M. Yarunov, Patent No. 2033520 (RU), Impact rotary mechanism, (20.04.1995)

32. Patent No. 2118454 (RU), Impact-turning mechanism, (27.08.1998).

33. L.T. Dvornikov, A.V. Nagibin, Patent No. 2362014 (RU), Multi-impact cam mechanism for drilling machines, (20.07.2009) 